\title{
Characteristics of Microstrip Fractal Antenna Shape Covered by Modified Split Ring Resonator
}

\author{
Amer Basim Shaalan ${ }^{1}$, Ammar Nadal Shareef ${ }^{2}$ \\ ' (Physics, College of Science/Al-Muthanna University, Iraq) \\ ${ }_{2}^{2}$ (Department of General Sciences, College of Basic Education/Al-Muthanna University, Iraq)
}

\begin{abstract}
Metamaterials exhibiting negative index of refraction has attracted a great amount of attention in recent years. These materials are artificial structures that exhibit characteristics not found in nature. Microstrip antennas covered by metamaterial are one of the interesting areas of study. In this paper fractal shape antenna is proposed and covered by two layers of modified split ring resonator. The results shows an enhancement in Reflection Coefficients, gain, directivity and far field shapes.
\end{abstract}

Keywords: Fractal Antenna, left handed materials, metamaterials, negative refractive index, split ring resonators

\section{Introduction}

Few years ago, there has been an interest in the study of Metamaterials. It is a class of composite materials, that is artificially constructed to exhibit properties not found in nature. Simultaneously negative permeability and permittivity over certain frequency range are the most important properties of interest. Veselago has studied this type of materials in 1968 [1]. He termed such media as left handed material (LHM) according to the direction of poyinting vector and $\mathrm{K}$ vector. He mentioned to some unique properties like negative refractive index and backward waves.

Microstrip patch antennas have become one of the most popular antennas because they have many advantages such as low-profile, light weight, low cost of fabrication. The addition of a superstrate layer over microstrip patch antenna (MPA) has been reported to enhance antenna gain and radiation efficiency [2]-[5]. In addition, superstrate layers are often used to protect microstrip patch antenna from its environment hazards, especially when mounted on aircrafts and missiles. Several configurations of superstrates were used to improve antenna radiation properties, such as dielectric slabs [6], electromagnetic bandgap (EBG) structures [7], highlyreflective surfaces [8], and the most recently artificial magnetic superstrates [9].

In this paper we introduce modified split ring resonator by adding more spaces to the open side that acts as capacitors, and use this design as a superstrate of two layers over fractal Quadruple Koch antenna shape. This configuration is shown in Fig.1. Ansoft's HFSS software was used to make simulation which is based on finite element method to solve S - parameters. Notable enhancement of gain and directivity obtained of this model of fractal shape metamaterial antenna.

\section{Split Ring Design}

Dimensions of the unit cell of split ring are $6 \mathrm{~mm}$ side length, $0.4 \mathrm{~mm}$ width of side, $0.4 \mathrm{~mm}$ width of the middle space and $0.2 \mathrm{~mm}$ width of extra spaces. We use three and five extra spaces in our design as shown in Fig.2. Arrangement of three different unit cells are simulate in a waveguide to calculate scattering parameters, meta-cover configuration is shown in Fig.3. Unit cells are etched on FR4 substrate of $\varepsilon_{\mathrm{r}}=4.4$ and $0.25 \mathrm{~mm}$ thickness. Relative permittivity, relative permeability and refractive index are determined using Nicolson-RossWeir approach [10 ].

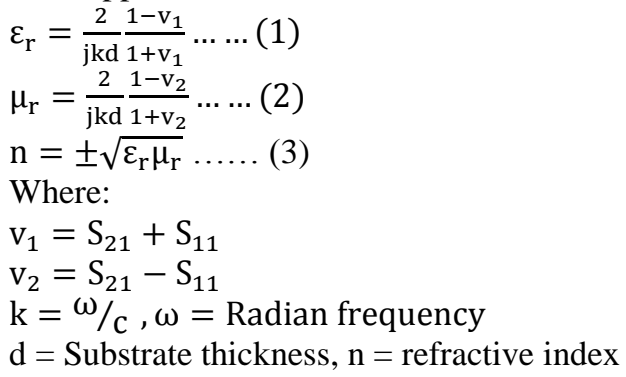




\section{Fractal Antenna Design}

In this section we will describe the fractal shape generation of the antenna and it's meta-cover. The starting shape of the antenna is a square of $28.5 \mathrm{~mm}$ side length. The generator is a second iteration of Koch curve applied to each side as shown in Fig.4. Details of Koch curve generating can be found in [11]. Patch antenna etched on substrate of dimensions $58 \mathrm{~mm} \times 58 \mathrm{~mm} \times 2 \mathrm{~mm}$. Material of substrate is FR4 with relative permittivity $\varepsilon_{\mathrm{r}}=4.4$.

\section{Results And Discussion}

Ansoft HFSS code is used here to design and simulate fractal antenna model covered by two layers of split ring metamaterial. We introduce different shapes of split ring, having three and five extra spaces. The resonance of each model is shown in Fig. 5, and the relation of each of relative permittivity, relative permeability and refractive index with the frequency is shown in Fig. 6. The refractive index can have negative values without the simultaneous negative values of $\mu^{\prime}$ and $\varepsilon^{\prime}$ if the condition, $\mu^{\prime} \varepsilon^{\prime \prime}+\mu^{\prime \prime} \varepsilon^{\prime}<0$ is satisfied [12]. This condition is plotted with frequency in Fig.7. Fig.5 shows that adding more spaces to the split ring will increase number of series capacitors and let the model resonate at higher frequency. In Fig.6, it is shown that the bandwidth; where refractive index negative is increased too.

Calculations of S-parameters of fractal antenna shape shows that this model has multi band behavior. It resonates at $5.76 \mathrm{GHz}, 6.4 \mathrm{GHz}$ and $7.32 \mathrm{GHz}$. This is shown in Fig.8. Gain and directivity of these three frequencies are listed in Table1. Shapes of radiation patterns are shown in Fig.9. By adding the two layers metacover to the antenna, we obtain enhancement of gain and directivity. Results are listed in Table 2. Fig.10 and Fig.11 shows $S_{11}$ and radiation pattern shape respectively of fractal antenna with meta-cover. Fig. 12 shows three dimensional far field of the antenna with and without meta-cover.

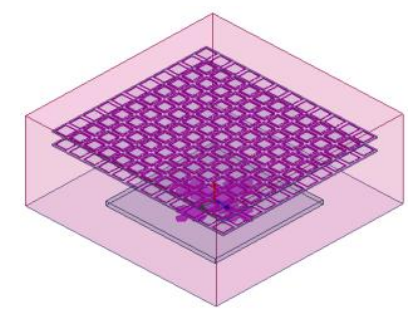

figure1. Configuration of Superstrate Over fractal Antenna Shape
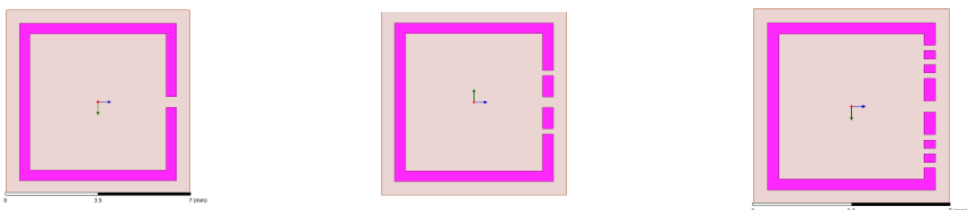

figure2. Modified Split Ring Resonator

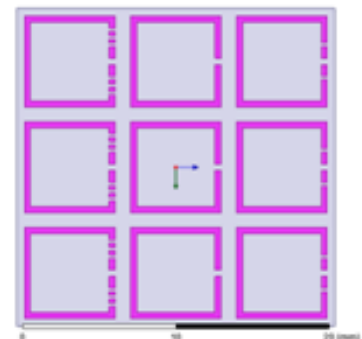

Figure3. Meta-Cover Configuration

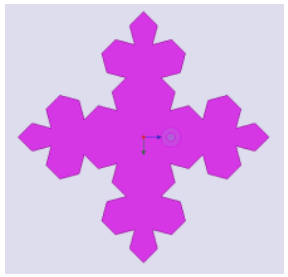

figure 4. Fractal Antenna Shape 


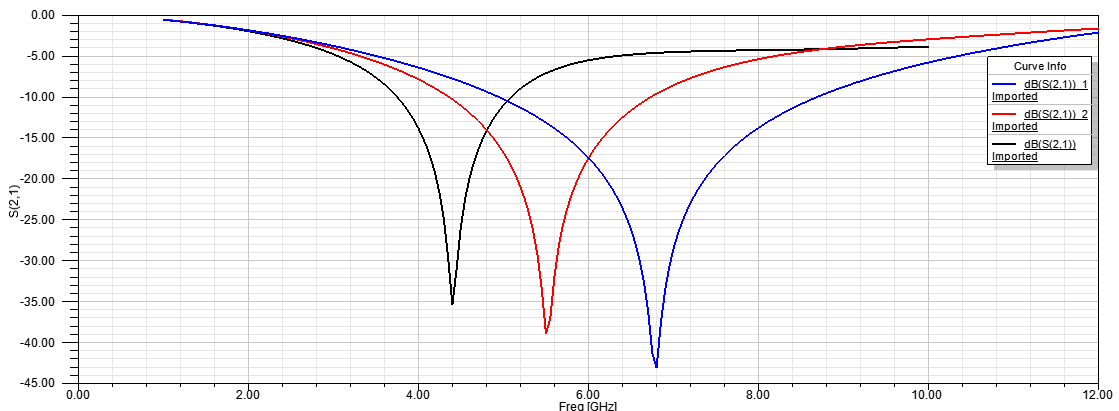

figure 5. $S_{21}$ Vs. Frequency of Split Ring of one, three and five spaces
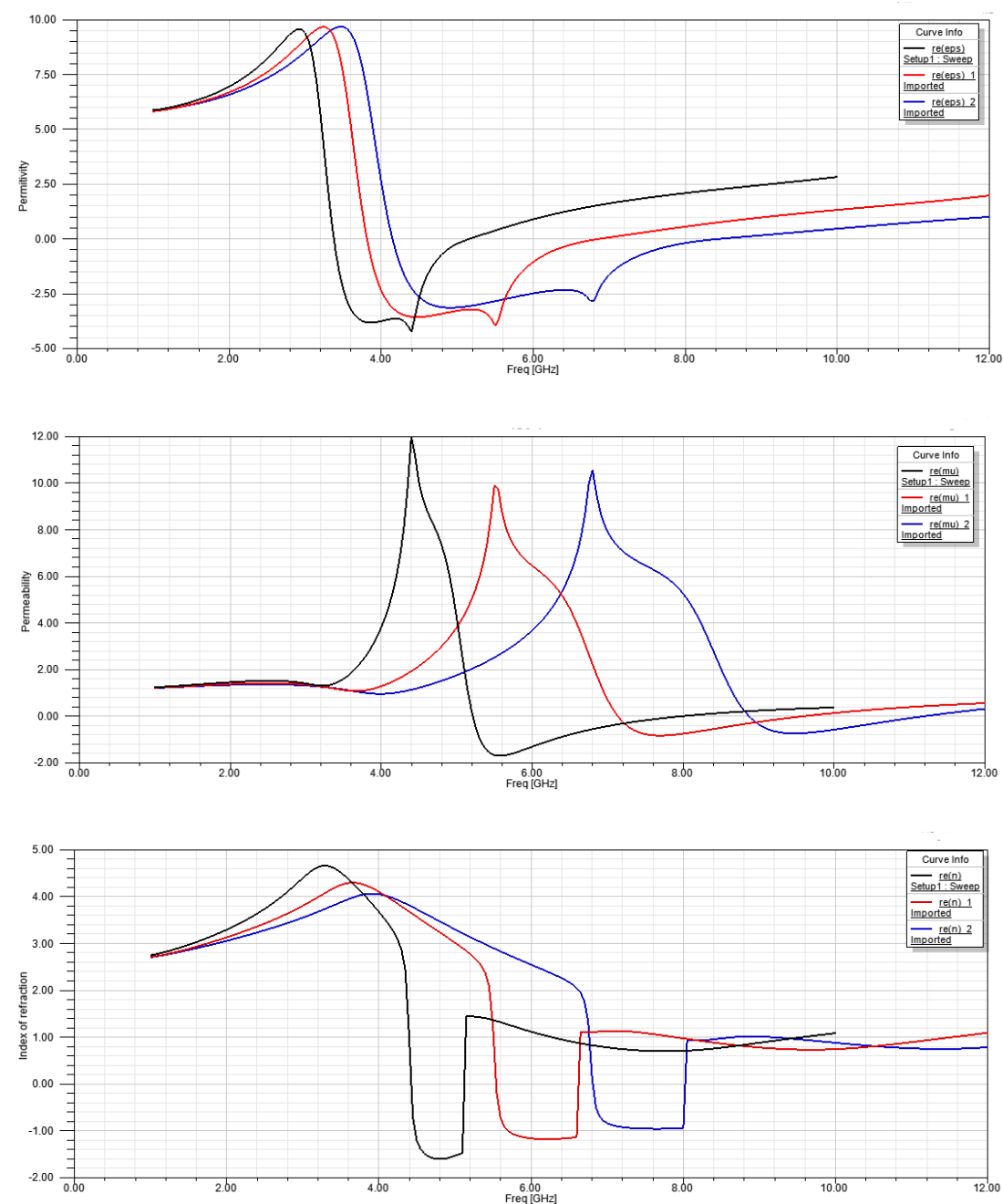

figure 6. Relative permittivity, Permeability, and Refractive index Vs. Freq.

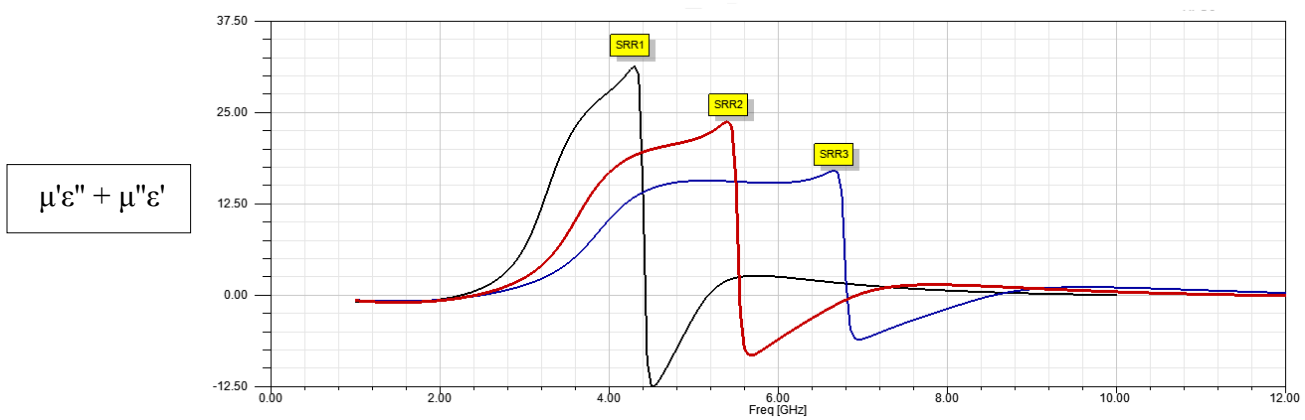

figure 7. $\mu^{\prime} \varepsilon^{\prime \prime}+\mu^{\prime \prime} \varepsilon^{\prime}$ Vs. frequency 


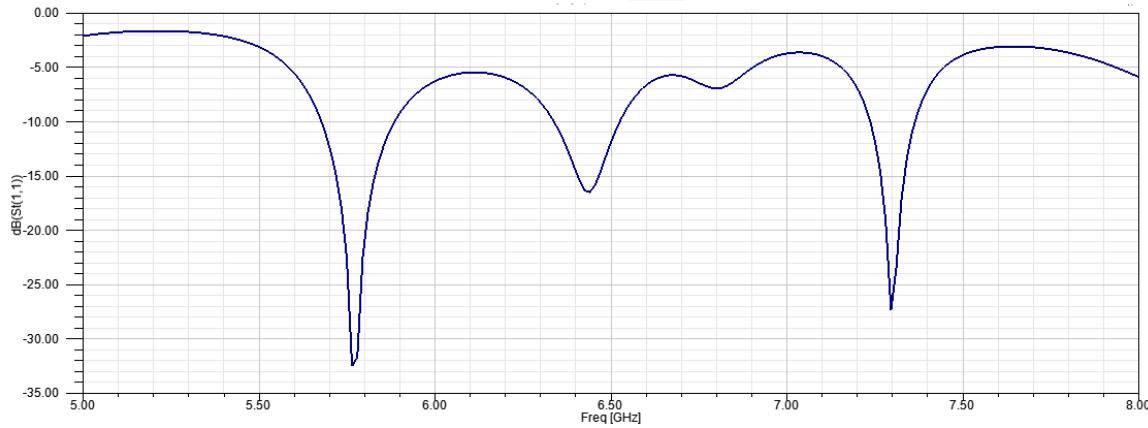

figure 8. $S_{11}$ Vs. Frequency of Fractal Antenna without meta-cover

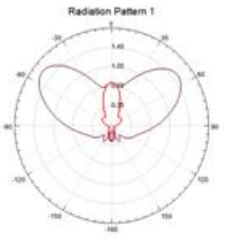

$\mathrm{f}=5.76 \mathrm{GHz}$

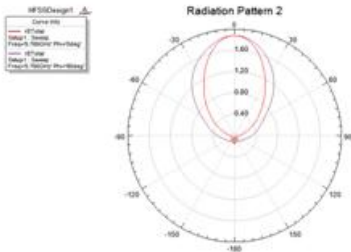

$\mathrm{f}=6.44 \mathrm{GHz}$

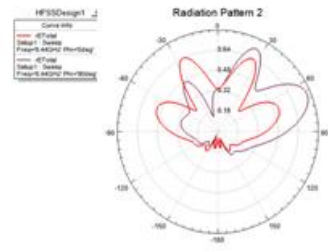

$\mathrm{f}=7.3 \mathrm{GHz}$

figure 9. Radiation Pattern of Fractal Antenna without meta-cover

Table 1. Fractal Antenna Parameters without meta-cover

\begin{tabular}{|c|c|c|c|}
\hline Frequency $(\mathrm{GHz})$ & Gain $(\mathrm{dB})$ & Directivity $(\mathrm{dB})$ & S11 $(\mathrm{dB})$ \\
\hline 5.76 & 6.1 & 8.9 & -32.4 \\
\hline 6.4 & 7.7 & 10.4 & -16.4 \\
\hline 7.29 & 2.1 & 8.1 & -27 \\
\hline
\end{tabular}

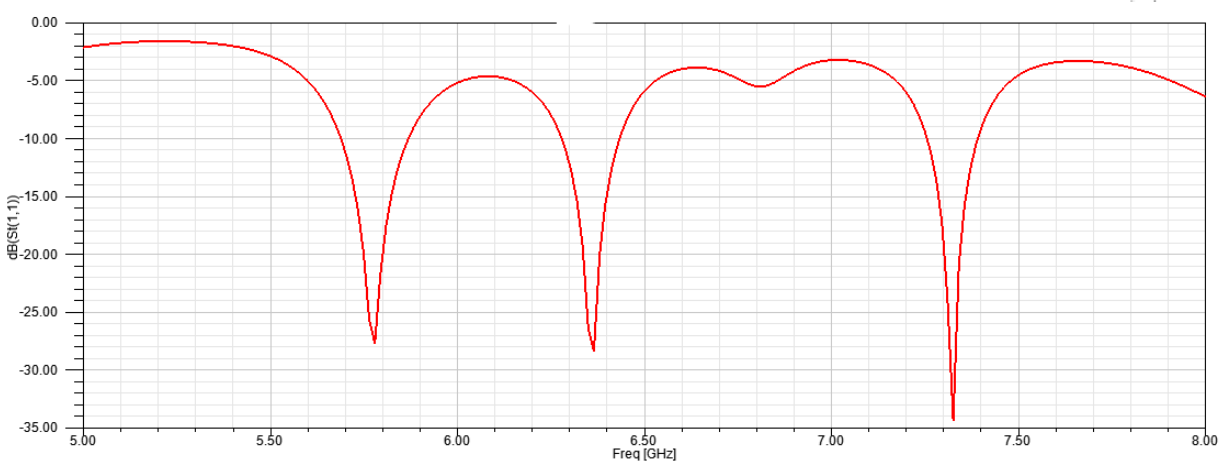

figure 10. $S_{11}$ Vs. Frequency of Fractal Antenna with meta-cover

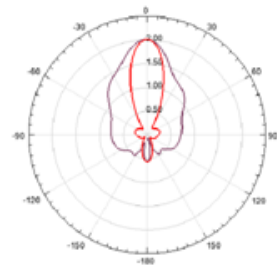

$\mathrm{f}=5.76 \mathrm{GHz}$

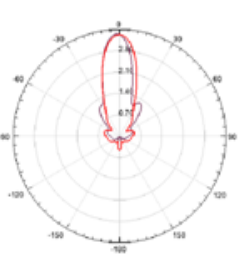

$\mathrm{f}=6.48 \mathrm{GHz}$

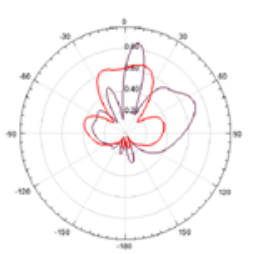

$\mathrm{f}=7.32 \mathrm{GHz}$

figure 11. Radiation Pattern of Fractal Antenna with meta-cover

Table 2. Fractal Antenna Parameters with meta-cover

\begin{tabular}{|c|c|c|c|}
\hline Frequency $(\mathrm{GHz})$ & Gain $(\mathrm{dB})$ & Directivity $(\mathrm{dB})$ & S11 $(\mathrm{dB})$ \\
\hline 5.76 & 8.24 & 11.1 & -27 \\
\hline 6.48 & 12.9 & 15.3 & -29 \\
\hline 7.32 & 2.4 & 8.3 & -34 \\
\hline
\end{tabular}




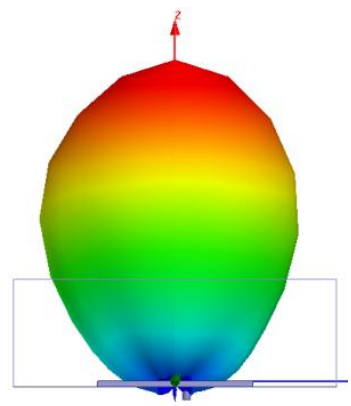

(a)

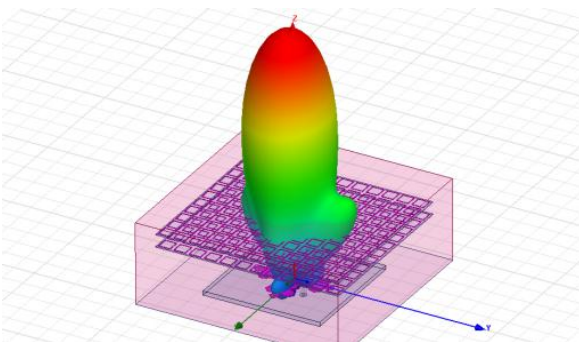

(b)

figure12. Three Dimensional Far Field Antenna (a) without meta-cover (b) with meta-cover

\section{Conclusion}

In this paper we use modified split ring resonator as two layers meta-cover over fractal quadruple Koch shape antenna. Distance between antenna and cover is $25 \mathrm{~mm}$. The two layers are separated by $4 \mathrm{~mm}$. Results shows best enhancement in gain and directivity at $25 \mathrm{~mm}$ distance between antenna and meta-cover. There is an enhancement and smoothen in far field shape of the antenna. This enhancement of antenna parameters is related to the extra spaces added to the unit cell of meta-cover.

This model is very useful in point to point communication and meta-cover can protect the antenna from environment hazards in addition to enhance antenna parameters.

\section{References}

[1]. V. G. Veselago, Electrodynamics of substrates with simultaneously negative values of $\varepsilon$ and $\mu$, Sov. Phys. Usp., Vol. 10, No. 4, 509-514, Apr. 1968

[2]. Wu, B-I, W. Wang, J. Pacheco, X. Chen, T. Grzegorczyk, and J.A. Kong, A study of using metamaterials as antenna substrate to enhance gain, Progress in Electromagnetic Research, PIERS 51, pp. 295-328, 2005.

[3]. B. Garg, R. Tiwari, A. Kumar and T. Chitransh, Design of factored ' $X$ ' shaped metamaterial structure for enhancement of patch antenna gain, International Conference on Communication Systems and Network Technologies 2011

[4]. D. Sarkar, K. Saurav, and K. Vaibhav Srivastava, Design of a Novel Dual-band Microstrip Patch Antenna for WLAN/WiMAX Applications Using Complementary Split Ring Resonators and Partially Defected Ground Structure, Progress In Electromagnetics Research Symposium proceedings, Taipei, March 25-28, 2013

[5]. A. Foroozesh and L. Shafai, Effects of artificial magnetic conductors in the design of low-profile high-gain planar antennas with high permittivity dielectric superstrate, IEEE Antenna Wireless Propagat.Lett., vol. 8, pp. 10 -13, 2009

[6]. H. Vettikalladi, O. Lafond, and M. Himdi, High-efficient and high-gain superstrate antenna for 60-ghz indoor communication, IEEE Antenna Wireless Propagat. Lett., vol. 8, pp. 1422-1425, 2009

[7]. H. Attia and O. M. Ramahi, EBG superstrate for gain and bandwidth enhancement Of microstrip array antennas, in Proceeding of IEEE AP-SInt. Symp. Antennas Propagation, San Diego, CA, pp. 1-4, 2008

[8]. A. Foroozesh and L. Shafai, Investigation into the effects of the patch type fss superstrate on the high-gain cavity resonance antenna design, IEEE Trans. Antennas Propagat., vol. 58, no. 2, pp. 258-270, Feb. 2010

[9]. H. Attia, L. Yousefi, M. M. Bait-Suwailam, M. S. Boybay, and O. M.Ramahi, Enhanced-gain microstrip antenna using engineered magnetic superstrates, IEEE Antenna Wireless Propagat. Lett., vol. 8, pp. 1198-1201, 2009

[10]. R. W. Ziolkwski, Design, fabrication, and testing of double negative metamaterials, IEEE Transactions on Antennas and Wireless Propagation, Vol.51, No. 7, 1516-1529, 2003

[11]. C. Puente, J. Romeu, R. Pous, J. Ramis and A. Hijazo, Small but Long Koch Fractal Monopole, Electronics Letters, Vol. 34, No. 1, pp. $9-10,1998$

[12]. T. T. Nguyen, P. Lievens1, Y. P. Lee and D. L. Vu, Computational studies of a cut-wire pair and combined metamaterials. Advances in Natural Sciences: Nanoscience and Nanotechnology. Vol. 2, No. 3, p: 9. 2011 